\title{
Efficacy and safety of weekly nab-paclitaxel plus gemcitabine in Chinese patients with metastatic adenocarcinoma of the pancreas: a phase II study
}

Ruihua $\mathrm{Xu}^{1}$, Xianjun $\mathrm{Yu}^{2}$, Jihui Hao ${ }^{3}$, Liwei Wang ${ }^{4}$, Hongming Pan ${ }^{5}$, Guohong Han ${ }^{6}$, Jianming $\mathrm{Xu}^{7}$, Yanqiao Zhang ${ }^{8}$, Shujun Yang ${ }^{9}$, Jia Chen ${ }^{10}$, Jieer Ying ${ }^{11}$, Guanghai Dai ${ }^{12}$, Mingyu Li ${ }^{13}$, Damir Begic ${ }^{13}$, Brian Lu ${ }^{13}$ and Lin Shen ${ }^{14,15^{*}}$

\begin{abstract}
Background: This phase II bridging study assessed the safety and efficacy of nab-paclitaxel/gemcitabine (Metastatic Pancreatic Adenocarcinoma Clinical Trial [MPACT] regimen) in Chinese patients with metastatic pancreatic cancer (MPC).

Methods: This 3-part sequential study evaluated nab-paclitaxel $125 \mathrm{mg} / \mathrm{m}^{2}$ plus gemcitabine $1000 \mathrm{mg} / \mathrm{m}^{2}$ on days 1,8 , and 15 every 4 weeks. Part 1 evaluated safety. Part 2 evaluated efficacy using Simon's optimal 2-stage design: if $>2$ responses were observed in Stage $1(n=28), 54$ additional patients would be enrolled in Stage 2. If $>9$ responses were observed, the study was complete. Otherwise, nab-paclitaxel/gemcitabine would be compared with gemcitabine alone in Part 3. The primary endpoint was overall response rate (ORR). Secondary endpoints included duration of response (DOR), overall survival (OS), and safety.
\end{abstract}

Results: Eighty-three patients were treated. The prespecified primary endpoint was met: the independently assessed ORR in Stages $1+2$ was 35\% (95\% Cl, 24.8-46.2); therefore, Part 3 was not initiated. The median DOR was 8.9 months (95\% Cl, 6.01-8.94). The median OS and progression-free survival were $9.2(95 \% \mathrm{Cl}, 7.6-11.1)$ and $5.5(95 \% \mathrm{Cl}, 5.29-7.16)$ months, respectively. The 12-month OS rate was 30\%. In an updated analysis, the median OS was 9.3 months and the 12-month OS rate was 32\%. Longer OS was observed in patients with baseline neutrophil-to-lymphocyte ratio $\leq 5 \mathrm{vs}>5$. The most common grade $\geq 3$ adverse events were leukopenia (35\%), neutropenia (34\%), anemia (15\%), thrombocytopenia (10\%), and fatigue (13\%). Grade 3 peripheral neuropathy occurred in 7\% of patients (no grade 4 reported).

Conclusions: The MPACT regimen of nab-paclitaxel/gemcitabine is efficacious in Chinese patients with MPC. No new safety signals were observed.

Trial registration: NCT02135822, May 8, 2014.

Keywords: nab-paclitaxel, Gemcitabine, MPACT, Pancreatic cancer, Metastatic, Chinese

\section{Background}

Pancreatic cancer is a growing health problem in China, where, similar to global trends, mortality nearly equals incidence [1, 2]. Epidemiological data from China's National Cancer Center Registry estimate that 79,400

\footnotetext{
* Correspondence: linshenpku@163.com

${ }^{14}$ Peking University Cancer Hospital and Institute, No. 52 Fucheng Road, Haidian District, Beijing 100142, China

${ }^{15}$ Department of Gastrointestinal Oncology, Peking University Cancer Hospital and Institute, No. 52 Fucheng Road, Haidian District, Beijing 100142, China

Full list of author information is available at the end of the article
}

people died from this disease in 2015 [3]. However, because these data are collected from multiple populationbased cancer registries, they represent a small portion of the Chinese national population and may underestimate the true burden of pancreatic cancer. Similarly, a paucity of survival data exists for Chinese patients. A recent study from the Shanghai Cancer Registry reported a 5 -year overall survival (OS) rate of $4.1 \%$ for all stages and tumor grades analyzed [4]. In China, approved treatment options for metastatic pancreatic cancer (MPC) are limited. 
In the European Union and the United States, nab-paclitaxel in combination with gemcitabine has received approval for the first-line treatment of $\operatorname{MPC}[5,6]$. This approval was based on the global phase III Metastatic Pancreatic Adenocarcinoma Clinical Trial (MPACT), in which first-line nab-paclitaxel/gemcitabine treatment demonstrated a significantly better OS and overall response rate (ORR) than did gemcitabine alone in 861 patients from North America, Europe, and Australia [7, 8]. The combination of nab-paclitaxel/gemcitabine is also recommended for first-line treatment of patients with MPC by the National Comprehensive Cancer Network guidelines, which are often followed by Chinese physicians [9]. nab-Paclitaxel/gemcitabine may also be a suitable first-line treatment regimen for Chinese patients with MPC, despite known differences in cancer drug tolerability between Asian and white populations [10]. These differences may result from genetic or environmental factors, among other things, and one of the most commonly reported examples is increased chemotherapy-induced myelosuppression in Asian vs white patients [11-13]. Based on clinical trials in metastatic breast cancer, the safety profile of nab-paclitaxel monotherapy appears largely similar between Western and Chinese populations [14, 15]. However, limited data exist on the safety and tolerability of nab-paclitaxel/gemcitabine in Chinese patients. A phase I/II study evaluated this combination in Chinese patients with advanced pancreatic cancer, albeit at a dose and schedule different from that administered in MPACT [7, 8, 16]. Although the study did not meet its primary endpoint of identifying the maximum tolerated dose in Chinese patients, $n a b$-paclitaxel $120 \mathrm{mg} / \mathrm{m}^{2}$ (the highest dose tested) plus gemcitabine $1000 \mathrm{mg} / \mathrm{m}^{2}$ on days 1 and 8 every 3 weeks was the recommended dosage/ schedule for these patients. With respect to dose intensity, this regimen was comparable with the MPACT regimen and resulted in a tolerable safety profile $[7,16]$.

In this phase II study, the efficacy and safety of the $n a b$-paclitaxel/gemcitabine regimen used in the MPACT study were evaluated in Chinese patients with MPC.

\section{Methods}

\section{Study Population}

Patients with histologically or cytologically confirmed metastatic pancreatic adenocarcinoma measurable by Response Evaluation Criteria in Solid Tumors (RECIST) version 1.0 were enrolled in this study. Key eligibility requirements included $\geq 18$ years of age, no prior treatment for metastatic disease, Karnofsky performance status $(\mathrm{KPS}) \geq 70$, and adequate hematologic, renal, and liver function. Patients with known brain metastases or baseline peripheral neuropathy grade $\geq 2$ were excluded.

This study was conducted in accordance with the Declaration of Helsinki and Good Clinical Practice Guidelines of the International Conference on
Harmonisation. Informed consent was obtained from all patients prior to study entry. The trial is registered at ClinicalTrials.gov (NCT02135822).

\section{Study Design}

This phase II, multicenter, 3-part sequential study was conducted at 13 sites in China. Part 1 evaluated the dose of nab-paclitaxel/gemcitabine based on safety. In Part 1, 10 patients were to be enrolled and treated with nab-paclitaxel $125 \mathrm{mg} / \mathrm{m}^{2}$ intravenously (IV) plus gemcitabine $1000 \mathrm{mg} /$ $\mathrm{m}^{2}$ IV once weekly for 3 weeks followed by a week of rest (qw 3/4). Safety data were evaluated after the last enrolled patient completed 2 treatment cycles or earlier if treatment was not tolerable or when $\geq 66 \%$ of patients tolerated $\geq 2$ treatment cycles without dose delay or modification. If it was determined in Part 1 that nab-paclitaxel $125 \mathrm{mg} / \mathrm{m}^{2}$ was the recommended dose for Part 2, the 10 patients from Part 1 were counted as a portion of the Part 2 enrollment. If the initial dose level in Part 1 was not tolerated, the Part 2 starting doses were to be reduced to nab-paclitaxel $100 \mathrm{mg} / \mathrm{m}^{2}$ plus gemcitabine $800 \mathrm{mg} / \mathrm{m}^{2}$.

Part 2 evaluated the efficacy of $n a b$-paclitaxel/gemcitabine based on a single-arm, Simon's optimal 2-stage design [17]. Patients in Part 2 were treated with the nabpaclitaxel and gemcitabine dose levels selected from Part 1. In Stage 1, the planned enrollment was 28 patients. If $>2$ responses were observed, an additional 54 patients would be enrolled in Stage 2 for treatment at the same dose level. In Stage 2, if $>9$ of 82 responses were observed, the study would be complete. If an insufficient number of responses was observed after Stage 1 or Stage 2 , the study would progress to Part 3.

Part 3 was designed to evaluate the efficacy and safety of $n a b$-paclitaxel/gemcitabine vs gemcitabine alone based on a randomized 2-arm design. Planned total enrollment for Part 3 was 154 patients. Patients were to be randomized 1:1 to receive the Part 1 recommended dose of $n a b$-paclitaxel followed by gemcitabine on days $1,8,15,29,36$, and 43 or gemcitabine $1000 \mathrm{mg} / \mathrm{m}^{2}$ IV alone weekly for 7 of 8 weeks (cycle 1). Subsequent treatments in both arms would occur on days 1, 8, and 15 of a 28-day cycle. Randomization would be stratified by liver metastasis and KPS score.

\section{Study Assessment}

The primary endpoint of the study was independently assessed ORR according to RECIST 1.0. Secondary endpoints included duration of response (DOR) according to RECIST 1.0, OS, safety, and tolerability. Exploratory endpoints were disease control rate (the percentage of patients achieving objective tumor response or stable disease for $\geq 16$ weeks), serum carbohydrate antigen 19-9 levels and potential association with clinical outcomes, patient-reported quality of life using the European Organisation of Research and Treatment of Cancer Quality of 
Life Questionnaire-Core 30, and tumor biomarker analysis. Ad hoc analyses included progression-free survival (PFS) and potential association of baseline neutrophil-tolymphocyte ratio (NLR) and OS. Efficacy was evaluated in the intent-to-treat population, which included all enrolled patients. Response and progression were independently assessed by a central imaging reviewer, blinded to treatment, according to radiological review by computed tomography scan or magnetic resonance imaging every 8 weeks per RECIST 1.0. Treatment continued until unacceptable toxicity or disease progression. Safety was assessed on days $1,8,15$, and 22 of each cycle by the investigator in all patients who received $\geq 1$ dose of study drug. Adverse events (AEs) were classified by the Medical Dictionary for Regulatory Activities version 17.0 system, and severity was evaluated according to the National Cancer Institute's Common Terminology Criteria for Adverse Events version 3.0. Dose reductions, delays, premature discontinuations, and clinical laboratory data were also evaluated.

\section{Sample Size and Statistical Analysis}

In Part 2, Simon's optimal 2-stage design was used. The 1sided hypothesis test on the ORR was $\mathrm{H}_{0}$ : ORR $\leq 7 \%$ vs $\mathrm{H}_{1}$ : ORR $\geq 19 \%$. The hypotheses were based on the ORR results from MPACT; the observed ORR was 23\% (2-sided 95\% CI, 19\%-27\%) for the nab-paclitaxel/gemcitabine arm and 7\% (2-sided 95\% CI, 5\%-10\%) for gemcitabine alone. The planned sample size of 82 patients was estimated to provide $90 \%$ power at a 1 -sided significance level of 0.05 [7]. The primary endpoint was analyzed based on the exact binomial distribution, and a 2-sided 95\% CI was estimated using the Clopper-Pearson method. DOR, OS, OS by baseline NLR (cutoffs $=5$ and median value), and PFS were analyzed by the Kaplan-Meier method. The data cutoff date was 1 June 2015. Data obtained using a cutoff date of 9 June 2016 were analyzed to determine updated OS rates. For the OS by baseline NLR subgroup analysis, the hazard ratio (HR) and 2-sided 95\% CI were estimated using the nonstratified Cox proportional hazard model, and the survival distributions for the 2 baseline NLR groups were compared using the nonstratified log-rank test.

\section{Results}

\section{Patients}

In total, 83 patients were enrolled in Part 2. The baseline characteristics are described in Table 1 . The median age was 57.0 years, and $19 \%$ of patients were aged $\geq 65$ years. Most patients (70\%) had a baseline KPS of 90 to 100 . The median baseline carbohydrate antigen 19-9 level for all patients was $602.8 \mathrm{U} / \mathrm{mL}$.

\section{Efficacy Results}

The initial dose administered to 15 patients in Part 1 was well tolerated; therefore, all patients in Part 2 were
Table 1 Baseline characteristics

\begin{tabular}{ll}
\hline Patient characteristics & $N=83$ \\
\hline Age, median (range), years & $57.0(30-78)$ \\
$\geq 65$ years, \% & 19 \\
Male, \% & 70 \\
KPS, \% & \\
$90-100$ & 70 \\
$70-80$ & 30 \\
Current site(s) of metastasis, \% & \\
Hepatic/liver & 83 \\
Abdomen/peritoneal & 53 \\
Lung/thoracic & 18 \\
No. of metastatic sites, \% & \\
1 & 35 \\
2 & 43 \\
3 & 19 \\
4 & 2 \\
CA 19-9, median (range), U/mL ${ }^{\text {a }}$ & 602.8 (0.93-1000) \\
Biliary stent, \% & 1 \\
\hline a CA 19-9 value above laboratory-defined upper limit of quantitation (1000 U/mL) is \\
listed as 1000 U/mL \\
CA 19-9 carbohydrate antigen 19-9, KPS Karnofsky performance status
\end{tabular}

treated with $n a b$-paclitaxel $125 \mathrm{mg} / \mathrm{m}^{2}$ plus gemcitabine $1000 \mathrm{mg} / \mathrm{m}^{2} \mathrm{qw} 3 / 4$. These 15 patients from Part 1 were included in Part 2. On the basis of combined results for Stages 1 and 2, the prespecified independently assessed ORR endpoint for Part 2 was met (35\%; 95\% CI, 24.8\%46.2\%; Table 2). Although no complete responses were observed, there were 29 (35\%) partial responses (PRs), and stable disease was achieved in 18 (22\%) patients. Thirteen (16\%) patients had progressive disease. The median DOR was 8.9 months (95\% CI, 6.01-8.94), and the disease control rate was 55\% (95\% CI, 44.1\%66.3\%; Table 2). Part 3 was not initiated per the study design (> 9 responses were observed in Part 2).

The median OS was 9.2 months (95\% CI, 7.6-11.1; Fig. 1), and the 1-year OS rate was 30\% (95\% CI, 14\%-47.6\%); 15\% of patients survived for $\geq 15$ months. The median follow-up for OS was 8.9 months (range, 0.7-15.1 months). In an updated analysis approximately 1 year later, the median OS was 9.3 months, with a median follow-up of 14.6 months (range, 0.7-21.7 months). The 12-month OS rate was $32 \%$ in the follow-up analysis.

Baseline NLR $\leq 5$ was associated with a longer OS vs NLR $>5$, although this difference was not significant (median, 10.0 vs 8.3 months; HR, 0.617; 95\% CI, $0.318-$ 1.197; $P=0.148$; Fig. 2). Because the $\mathrm{n}$ value in the $>5$ NLR arm was small $(n=23)$, a separate analysis using the median NLR baseline value (3.7) was performed; baseline NLR $\leq 3.7(n=42)$ vs $>3.7(n=41)$ was also associated with a longer OS, but the difference was not 
Table 2 Efficacy

\begin{tabular}{|c|c|}
\hline Outcome $^{a}$ & $\begin{array}{l}\text { nab-P + Gem } \\
N=83\end{array}$ \\
\hline ORR, $n(\%)$ & $29(35)$ \\
\hline$C R$ & 0 \\
\hline PR & $29(35)$ \\
\hline $\mathrm{SD}, n(\%)$ & $18(22)$ \\
\hline$P D, n(\%)$ & $13(16)$ \\
\hline Not evaluable, $n(\%)$ & $15(18)$ \\
\hline No postbaseline assessment, $n(\%)$ & $8(10)$ \\
\hline $\mathrm{DCR}, n(\%)^{\mathrm{b}}$ & $46(55)$ \\
\hline DOR, median $(95 \% \mathrm{Cl})$, months & $8.9(6.01-8.94)$ \\
\hline OS, median $(95 \% \mathrm{Cl})$, months & $9.2(7.6-11.1)$ \\
\hline \multicolumn{2}{|l|}{ OS rate, \% } \\
\hline 3 months & 89 \\
\hline 6 months & 70 \\
\hline 9 months & 53 \\
\hline 12 months & 30 \\
\hline 15 months & 15 \\
\hline Progression-free survival, median ( $95 \% \mathrm{Cl})$, months & $5.5(5.29-7.16)$ \\
\hline
\end{tabular}

$C R$ complete response, $D C R$ disease control rate, $D O R$ duration of response, Gem gemcitabine, nab-P nab-paclitaxel, ORR overall response rate, OS overall survival, $P D$ progressive disease, $P R$ partial response, $S D$ stable disease ${ }^{a}$ Percents may not add up to 100 due to rounding

${ }^{\mathrm{b}}$ Defined as the percentage of patients achieving objective tumor response or SD for $\geq 16$ weeks

significant (median, 10.0 vs 8.1 months; HR, 0.724; 95\% CI, 0.398-1.319; $P=0.288)$. The median PFS was 5.5 months (95\% CI, 5.29-7.16; Fig. 3).

\section{Treatment Exposure}

For all patients, the median duration of treatment was 4.8 months, and the median number of treatment cycles was 5 (range, 1-12). Forty-nine percent of patients had $\geq 1$ $n a b$-paclitaxel dose reduction, and $51 \%$ of patients had $\geq 1$ gemcitabine dose reduction, most due to AEs. The most common AEs leading to dose reduction of nab-paclitaxel and gemcitabine were thrombocytopenia (14\% and 20\%), neutropenia (14\% and 16\%), and leukopenia (11\% and $13 \%)$, respectively. At least 1 nab-paclitaxel or gemcitabine dose delay occurred in $37 \%$ of patients. The median cumulative doses of $n a b$-paclitaxel and gemcitabine were 1500 and $12,000 \mathrm{mg} / \mathrm{m}^{2}$, respectively. The median dose intensities of $n a b$-paclitaxel and gemcitabine were 79 and $627 \mathrm{mg} / \mathrm{m}^{2} /$ week, respectively. The median percentages of per-protocol dose of $n a b$-paclitaxel and gemcitabine were $85 \%$ and $84 \%$, respectively.

\section{Safety}

Seventy-five percent of patients experienced $\geq 1$ grade $\geq 3$ AE (Table 3). The most common grade $\geq 3$ AEs were leukopenia (35\%), neutropenia (34\%), anemia (15\%), thrombocytopenia (10\%), and fatigue (13\%). Grade 3 peripheral neuropathy occurred in only $7 \%$ of patients (no grade 4 reported). Twenty-four percent of patients reported $\geq 1$ serious treatment-emergent AE. Discontinuations due to AEs were relatively low (11\%).

\section{Discussion}

In this phase II study, the MPACT regimen (nab-paclitaxel $125 \mathrm{mg} / \mathrm{m}^{2}$ plus gemcitabine $1000 \mathrm{mg} / \mathrm{m}^{2}$ ) was efficacious and safe as first-line treatment of Chinese patients with MPC. Per protocol, the study did not progress to Part 3 because $>9$ responses were observed during Part 2 and the study was considered complete. Although no complete responses were observed in this study, $35 \%$ of patients had a partial response, and the median DOR was 8.9 months, indicating a durable response. The median OS was 9.2 months, and the OS rate at 1 year was $30 \%$ (9.3 months and $32 \%$, respectively, in an updated analysis). The regimen appeared to

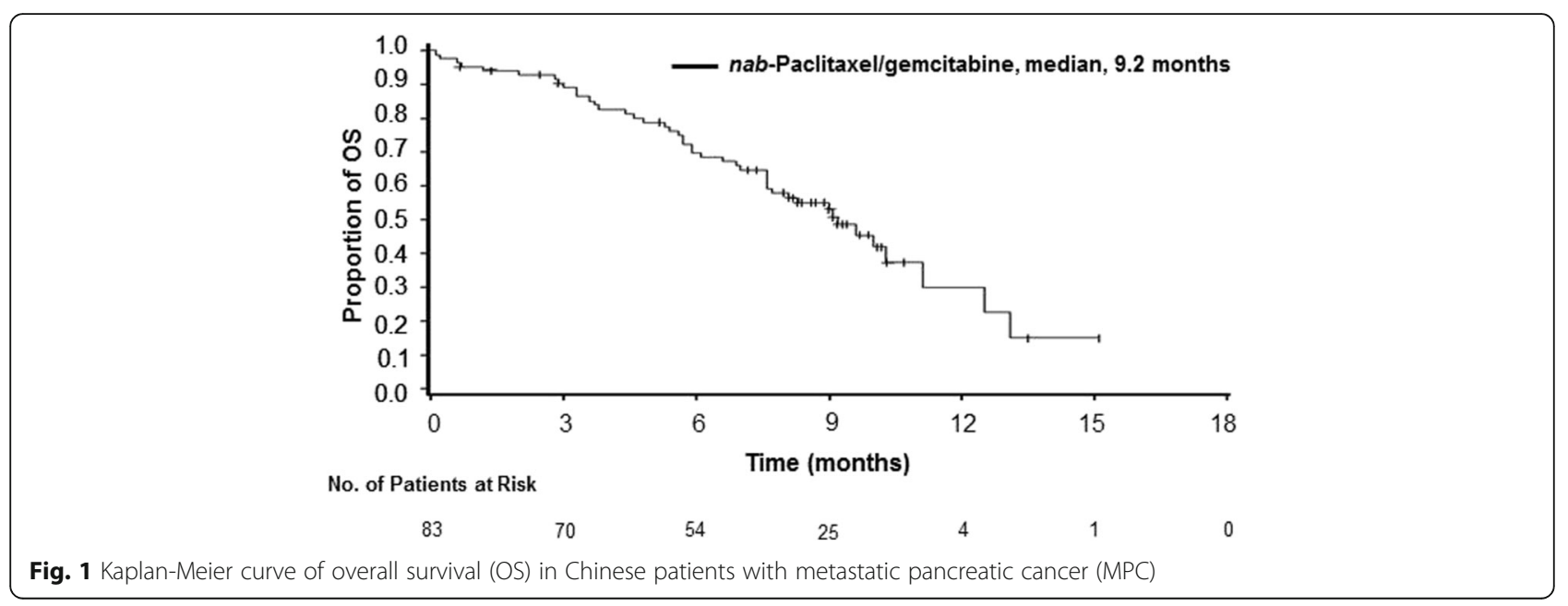




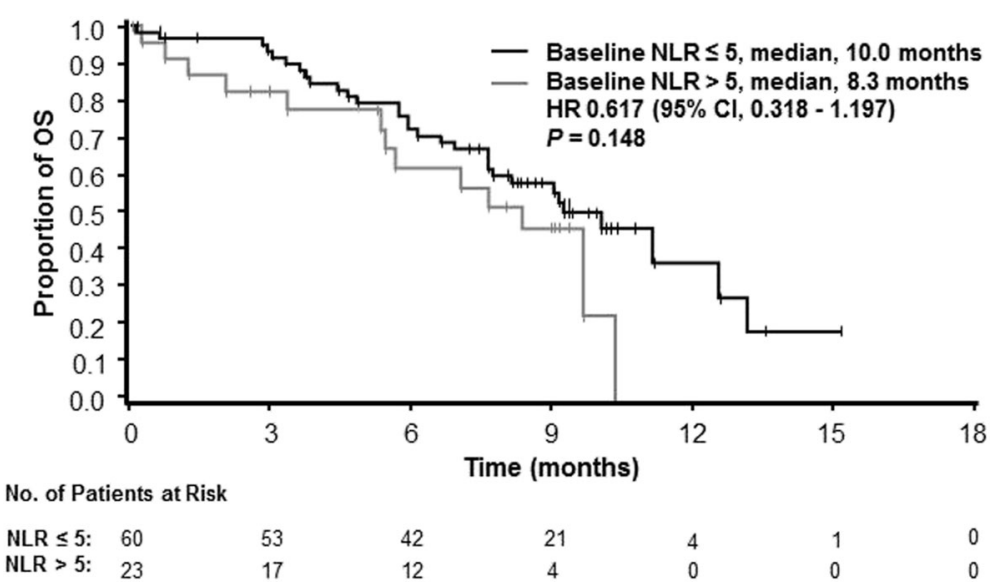

Fig. 2 Kaplan-Meier curve of overall survival by neutrophil-to-lymphocyte ratio (NLR) in Chinese patients with metastatic pancreatic cancer (MPC)

be well tolerated in Chinese patients with MPC, and no new safety signals were identified compared with those observed in the MPACT population [7].

Efficacy results in this study of Chinese patients were comparable with those reported in MPC trials using the same nab-paclitaxel/gemcitabine regimen in Western countries and Japan $[7,8,18]$. In the MPACT population, treatment with this nab-paclitaxel/gemcitabine regimen resulted in a median OS of 8.7 months compared with 9.2 months in the Chinese population (Table 4) [8]. Similar to the findings of MPACT, Chinese patients with a baseline NLR $\leq 5$ had a longer OS compared with those with a baseline NLR $>5$. The ORR was $23 \%$ in MPACT and $35 \%$ in the Chinese population, although treatment resulted in a slightly longer DOR in the global study (11.1 months in the MPACT population and 8.9 months in the Chinese population) [19]. In both populations, the median PFS was 5.5 months.

Although data from other studies of Chinese patients treated with $n a b$-paclitaxel/gemcitabine are limited, a phase I/II study evaluated 3 different doses of nabpaclitaxel $\left(80 \mathrm{mg} / \mathrm{m}^{2}, 100 \mathrm{mg} / \mathrm{m}^{2}\right.$, and $\left.120 \mathrm{mg} / \mathrm{m}^{2}\right)$ in combination with gemcitabine $1000 \mathrm{mg} / \mathrm{m}^{2}$, both given weekly for 2 weeks in a 21-day cycle in Chinese patients with advanced pancreatic cancer [16]. In that study, the maximum tolerated dose was not met; however, in the 12 patients treated with $n a b$-paclitaxel $120 \mathrm{mg} / \mathrm{m}^{2}$, the median OS and PFS were 12.2 and 5.2 months, respectively, and the ORR was $42 \%$. Similar to the findings in our study, common grade $3 / 4$ toxicities that were associated with the $120 \mathrm{mg} /$ $\mathrm{m}^{2}$ dose included neutropenia (17\%) and thrombocytopenia (8\%), and grade $3 / 4$ sensory neuropathy occurred in only 1 patient. In a trial of Japanese patients with MPC, outcomes of treatment with the nab-paclitaxel/gemcitabine MPACT regimen were also higher/longer compared with the outcomes in the MPACT population [18]. These findings further support the use of the MPACT regimen for the treatment of Asian patients with MPC.

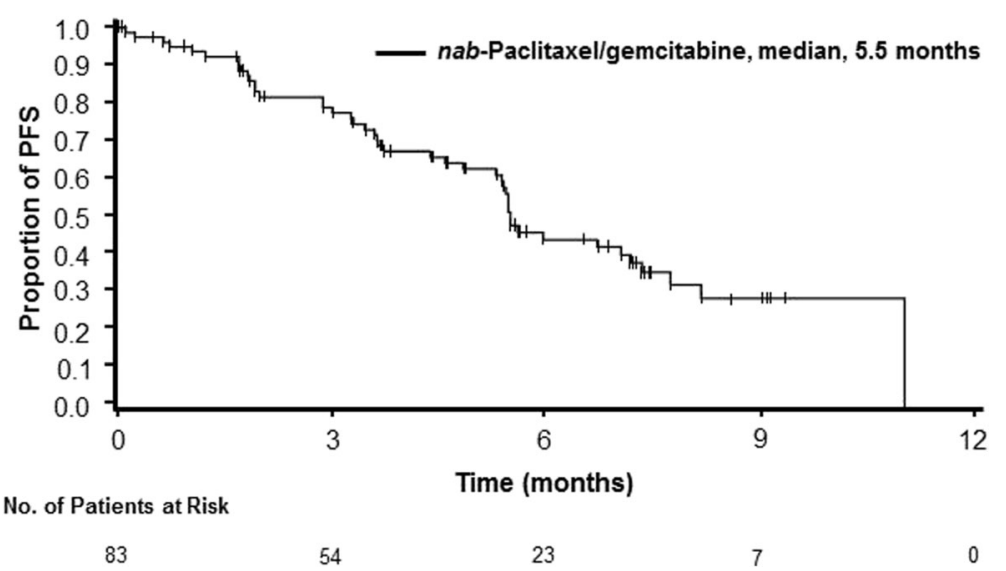

Fig. 3 Kaplan-Meier curve of progression-free survival (PFS) in Chinese patients with metastatic pancreatic cancer (MPC) 
Table 3 Grade $\geq 3$ treatment-emergent adverse events in $\geq 10 \%$ of patients

\begin{tabular}{ll}
\hline Grade $\geq 3$ adverse events, $n(\%)$ & $\begin{array}{l}n a b-P+G e m \\
N=83\end{array}$ \\
\hline Pts with at least 1 grade $\geq 3$ AE & $62(75)$ \\
Hematologic AEs $^{\mathrm{a}}$ & \\
Leukopenia & $28(35)$ \\
Neutropenia & $27(34)$ \\
Anemia & $12(15)$ \\
Thrombocytopenia & $8(10)$ \\
Nonhematologic AEs & \\
Fatigue & $11(13)$ \\
\hline
\end{tabular}

$A E$ adverse event, Gem gemcitabine, nab-P nab-paclitaxel

${ }^{\mathrm{a} B}$ Based on laboratory values; $n=80$ patients assessed

In the current study, the most common treatmentemergent grade $\geq 3$ AEs were leukopenia, neutropenia, anemia, thrombocytopenia, and fatigue. Similarly, the most common grade $\geq 3$ AEs in MPACT were neutropenia, leukopenia, thrombocytopenia, anemia, fatigue, and peripheral neuropathy [7]. The incidence of peripheral neuropathy was one noteworthy difference between these two trials. In the MPACT population, $17 \%$ of patients experienced grade $\geq 3$ peripheral neuropathy compared with only $7 \%$ of Chinese patients in this study. The definitive reasons for this are unclear, and many factors, such as ethnic differences or regional variations in treatments for neuropathy, could be involved [20, 21]; this would be an interesting topic to investigate in the future. In addition, nab-paclitaxel treatment modifications due to AEs were less frequent in the MPACT population compared with the Chinese population [7]. nab-Paclitaxel dose reductions occurred in $41 \%$ and $49 \%$ of patients in MPACT and the Chinese study, respectively.

Table 4 Efficacy Outcomes of nab-paclitaxel plus gemcitabine in MPACT and the Chinese study

\begin{tabular}{|c|c|c|}
\hline Parameter & MPACT $[7,8,19]$ & Chinese Study \\
\hline$n$ & 431 & 83 \\
\hline OS, median, months & 8.7 & 9.2 \\
\hline$N L R \leq 5$ & 9.1 & 10.0 \\
\hline$N L R>5$ & 5.0 & 8.3 \\
\hline PFS, median, months ${ }^{a}$ & 5.5 & 5.5 \\
\hline ORR, $\%^{\mathrm{a}}$ & 23 & 35 \\
\hline DCR, \% & 48 & 55 \\
\hline DOR, median, months & 11.1 & 8.9 \\
\hline
\end{tabular}

$D C R$ disease control rate, $D O R$ duration of response, NLR neutrophil-tolymphocyte ratio, MPACT Metastatic Pancreatic Adenocarcinoma Clinical Trial, ORR overall response rate, OS overall survival, PFS progression-free survival andependently assessed
Results from this phase II study in Chinese patients are positive; however, several factors must be considered to put the data in perspective. Although this was a bridging study to assess the safety and efficacy of nabpaclitaxel/gemcitabine in Chinese patients, one limitation was the homogeneous population. However, the impact of this limitation may have been addressed by the study's multicenter sampling. In addition, efficacy was evaluated based on a single treatment arm rather than on a comparison of outcomes between 2 randomized groups. The results described here in Chinese patients are similar to those of the global MPACT study, though cross-trial comparisons should be interpreted with caution because of differences in factors such as patient population and usual supportive care. For example, in our study, a higher percentage of Chinese patients had a better baseline performance status (KPS of 90-100) than patients in the global MPACT population $(70 \%$ vs $58 \%)$ [7]. Therefore, when comparing these 2 studies, it is possible that this difference could, in part, account for the improved efficacy outcomes observed in this study compared with the MPACT study. Further, although only the first 2 parts of the 3-part study design were executed, the null hypothesis was rejected as more than 9 of the 82 patients (planned sample size) responded. Although Part 3 would have provided more rigor to the overall statistical testing of nab-paclitaxel/ gemcitabine vs gemcitabine in this disease setting, it would only have been triggered if sufficient activity was not observed vs known historical data in Part 2. Such adaptive trial designs are generally more efficient, requiring fewer patients to answer research questions. This unique study design was particularly beneficial and relevant in this disease setting and helped to avoid enrolling Chinese patients into an inferior treatment arm, as a large global study has established the significant clinical benefit of $n a b$-paclitaxel/gemcitabine vs gemcitabine.

\section{Conclusion}

The nab-paclitaxel/gemcitabine regimen used in MPACT was efficacious and well tolerated in Chinese patients with MPC, supporting the use of this combination regimen in this patient population.

\footnotetext{
Abbreviations

AE: adverse event; DOR: duration of response; HR: hazard ratio; IV: intravenously; KPS: Karnofsky performance status; MPACT: Metastatic Pancreatic Adenocarcinoma Clinical Trial; MPC: metastatic pancreatic cancer; NLR: neutrophil-to-lymphocyte ratio; ORR: overall response rate; OS: overall survival; PFS: progression-free survival; PR: partial response; qw 3/4: the first 3 of 4 weeks; RECIST: Response Evaluation Criteria in Solid Tumors
}

\section{Acknowledgements}

The authors thank Richard Xue, Lotus Yung, and Xinyu Wei of Celgene Corporation for their support. Medical writing assistance was provided by Dena Jacob, PhD, of MediTech Media, funded by Celgene Corporation. The authors are fully responsible for all content and editorial decisions for this manuscript. 


\section{Funding}

Funding received from Celgene Corporation. Celgene was involved in the development of the protocol and analysis of the data. All authors, including those authors listed as employees of Celgene were involved in the writing, review, and provided final approval of the manuscript.

\section{Availability of data and materials}

Datasets were available to all authors and can be made available through Celgene's data sharing process upon reasonable request.

\section{Authors' contributions}

$M L, D B, B L$ analyzed and interpreted the patient data regarding all study endpoints and safety. RX, XY, JH, LW, HP, GH, JX, YZ, SY, JC, JY, GD, ML, DB, $\mathrm{BL}$, and $\mathrm{LS}$ collected data, were contributors to the writing of the manuscript, reviewed, and provided final approval of the manuscript.

\section{Authors' information}

Not applicable

\section{Ethics approval and consent to participate}

All relevant ethical approvals from institutional review board/independent ethics committee have been obtained prior to study commencement. Written informed consent was obtained from all patients prior to study entry. Institutional review boards/independent ethics committees included Ethics Committee of Beijing Cancer Hospital, Sun Yat-sen University Cancer Center Ethics Committee, Committee of Shanghai First People's Hospital, Jiangsu Cancer Hospital, Sir Run Run Shaw Hospital, College of Medicine, Zhejiang University, Shanghai Red Cross Cancer Hospital, Tianjin Cancer Institute and Hospital Ethics Committee, PLA General Hospital Ethics Committee, Affiliated Hospital of Academy of Military Medical Sciences, Xijing Hospital, Ethics Committee of Jiangsu Cancer Hospital, Harbin Medical University Cancer Hospital Ethics, and Ethics Committee of Zhejiang Cancer Hospital.

\section{Consent for publication}

Not applicable

\section{Competing interests}

$L S, R X, X Y, J H, L W, H P, G H, J X, Y Z, S Y, J C$, JY, and GD have nothing to disclose. ML, $\mathrm{DB}$, and $\mathrm{BL}$ are employees of and have stock ownership in Celgene Corporation.

\section{Publisher's Note}

Springer Nature remains neutral with regard to jurisdictional claims in published maps and institutional affiliations.

\footnotetext{
Author details

'Sun Yat-sen University Cancer Center, 651 Dongfeng East Road, Guangzhou 510060, China. ${ }^{2} F u d a n$ University Shanghai Cancer Center, No 270, Dongan Road, Shanghai 200032, China. ${ }^{3}$ Tianjin Cancer Hospital, Huan-Hu-Xi Road, Tianjin 300060, China. ${ }^{4}$ Renji Hospital, Shanghai Jiaotong University, 160 Pujian Lu, Shanghai 200127, China. ${ }^{5}$ Sir Run Run Shaw Hospital, Zhejiang University, 3 East Qingchun Road, Hangzhou City 310016, China. ${ }^{6}$ Xijing Hospital, W Rd, Xi'an, Changle 127, China. ${ }^{7} 307$ Hospital of the People's Liberation Army, Beijing 100021, China. ${ }^{8}$ Harbin Medical University Cancer Hospital, Haping Road No.150, Harbin, China. ${ }^{9}$ Henan Cancer Hospital, Zhengzhou 450003, China. ${ }^{10}$ Jiangsu Provincial Tumor Hospital, 300 Guangzhou Road, Nanjing 210029, China. ${ }^{11}$ Zhejiang Cancer Hospital, 38 Guangji Road, Banshan Bridge, Hangzhou City 310022, China. ${ }^{12}$ Chinese People's Liberation Army General Hospital No.28, Fuxing Road, Beijing, China ${ }^{13}$ Celgene Corporation, Summit, NJ, USA. ${ }^{14}$ Peking University Cancer Hospital and Institute, No. 52 Fucheng Road, Haidian District, Beijing 100142, China.

${ }^{15}$ Department of Gastrointestinal Oncology, Peking University Cancer Hospital and Institute, No. 52 Fucheng Road, Haidian District, Beijing 100142, China.
}

Received: 19 August 2016 Accepted: 8 December 2017 Published online: 22 December 2017

\section{References}

1. Lin QJ, Yang F, Jin C, Current FDL. Status and progress of pancreatic cancer in China. World J Gastroenterol. 2015;21(26):7988-8003.
2. World Health Organization. GLOBOCAN 2012: Estimated Cancer Incidence, Mortality and Prevalence Worldwide in 2012. http://globocan.iarc.fr/Pages/ fact_sheets_cancer.aspx. Accessed 17 Sept 2015.

3. Chen W, Zheng R, Baade PD, Zhang S, Zeng H, Bray F, et al. Cancer statistics in China, 2015. CA Cancer J Clin. 2016;66(2):115-32.

4. Luo J, Xiao L, Wu C, Zheng Y, Zhao N. The incidence and survival rate of population-based pancreatic cancer patients: shanghai cancer registry 2004-2009. PLoS One. 2013;8(10):e76052.

5. Abraxane for injectable suspension (paclitaxel protein-bound particles for injectable suspension) (albumin-bound). Summit, NJ: Celgene Corporation. 2015.

6. European Medicines Agency. Summary of opinion: Abraxane. http://www. ema.europa.eu/docs/en_GB/document_library/Summary_of_opinion/ human/000778/WC500155465.pdf. Accessed 21 Nov 2013.

7. Von Hoff DD, Ervin T, Arena FP, Chiorean EG, Infante J, Moore M, et al. Increased survival in pancreatic cancer with nab-paclitaxel plus gemcitabine. N Engl J Med. 2013;369(18):1691-703.

8. Goldstein D, El-Maraghi RH, Hammel P, Heinemann V, Kunzmann V, Sastre J, et al. Nab-paclitaxel plus gemcitabine for metastatic pancreatic cancer: long-term survival from a phase III trial. J Natl Cancer Inst. 2015;107(2) https://doi.org/10.1093/jnci/dju413.

9. NCCN Clinical Practice Guidelines in Oncology. Pancreatic Adenocarcinoma. V1.2016. Available at: http://www.nccn.org/professionals/physician_gls/f_ guidelines.asp\#site. Accessed 15 July 2012.

10. Ling WH, Lee SC. Inter-ethnic differences-how important is it in cancer treatment? Ann Acad Med Singap. 2011;40(8):356-61.

11. O'Donnell PH, Dolan ME. Cancer pharmacoethnicity: ethnic differences in susceptibility to the effects of chemotherapy. Clin Cancer Res. 2009;15(15):4806-14

12. Ma B, Yeo W, Hui P, Ho WM, Johnson PJ. Acute toxicity of adjuvant doxorubicin and cyclophosphamide for early breast cancer - a retrospective review of Chinese patients and comparison with an historic western series. Radiother Oncol. 2002;62(2):185-9.

13. Dattani N, Altham D, Coady K. Neutropenia in Asian patients with solid tumors receiving chemotherapy: a retrospective case-control study. J Solid Tumors. 2016:6(2):25-9.

14. Gradishar WJ, Tjulandin S, Davidson N, Shaw H, Desai N, Bhar P, et al. Phase III trial of nanoparticle albumin-bound paclitaxel compared with polyethylated castor oil-based paclitaxel in women with breast cancer. J Clin Oncol. 2005;23(31):7794-803.

15. Guan Z-Z, Li QL, Feng F, Jiang Z, Shen Z, Yu S, et al. Superior efficacy of a Cremophor-free albumin-bound paclitaxel compared with solvent-based paclitaxel in Chinese patients with metastatic breast cancer. Asia Pac J Clin Oncol. 2009;5(3):165-74

16. Zhang DS, Wang DS, Wang ZQ, Wang FH, Luo HY, Qiu MZ, et al. Phase I/I study of albumin-bound nab-paclitaxel plus gemcitabine administered to Chinese patients with advanced pancreatic cancer. Cancer Chemother Pharmacol. 2013;71(4):1065-72

17. Simon R. Optimal two-stage designs for phase II clinical trials. Control Clin Trials. 1989;10(1):1-10

18. Kasuga A, Ueno H, Ikeda M, Ueno M, Mizuno N, loka T, et al. Efficacy, safety and pharmacokinetics of weekly nab-paclitaxel plus gemcitabine in Japanese patients with metastatic pancreatic cancer (MPC): phase I/II trial. Presented at: APA/JPS 45th Anniversary Meeting; November 5-8, 2014; Big Island, HI, USA [abstr. 14200].

19. Shen L, Hao J, Wang L, Pan H, Han G, Xu J, et al. A phase II study of Chinese patients (pts) treated with nab-paclitaxel (nab-P) plus gemcitabine for metastatic pancreatic cancer (MPC). J Clin Oncol. 2016:34(4) [abstr. 327].

20. McQuade JL, Meng Z, Chen Z, Wei Q, Zhang Y, Bei W, et al. Utilization of and attitudes towards traditional Chinese medicine therapies in a Chinese cancer hospital: a survey of patients and physicians. Evid Based Complement Alternat Med. 2012:2012:504507.

21. Lee KH, Chang HJ, Han SW, DY O, Im SA, Bang YJ, et al. Pharmacogenetic analysis of adjuvant FOLFOX for Korean patients with colon cancer. Cancer Chemother Pharmacol. 2013;71(4):843-51. 